\title{
Formation and Reconnection of Three-Dimensional Current Sheets in the Solar Corona
}

\author{
J. K. Edmondson ${ }^{1,2}$, S. K. Antiochos ${ }^{1}$, C. R. DeVore ${ }^{3}$, and T. H. Zurbuchen ${ }^{2}$ \\ ${ }^{1}$ NASA Goddard Space Flight Center, Greenbelt, MD 20771 \\ ${ }^{2}$ Dept of Atmospheric, Oceanic, and Space Science, University of Michigan, Ann Arbor, \\ MI 48105 \\ ${ }^{3}$ Naval Research Laboratory, Washington, DC 20375 \\ jkedmond@umich.edu
}




\begin{abstract}
Current-sheet formation and magnetic reconnection are believed to be the basic physical processes responsible for much of the activity observed in astrophysical plasmas, such as the Sun's corona. We investigate these processes for a magnetic configuration consisting of a uniform background field and an embedded line dipole, a topology that is expected to be ubiquitous in the corona. This magnetic system is driven by a uniform horizontal flow applied at the line-tied photosphere. Although both the initial field and the driver are translationally symmetric, the resulting evolution is calculated using a fully three-dimensional magnetohydrodynamic (3D MHD) simulation with adaptive mesh refinement that resolves the current sheet and reconnection dynamics in detail. The advantage of our approach is that it allows us to apply directly the vast body of knowledge gained from the many studies of $2 \mathrm{D}$ reconnection to the fully $3 \mathrm{D}$ case. We find that a current sheet forms in close analogy to the classic Syrovatskii 2D mechanism, but the resulting evolution is different than expected. The current sheet is globally stable, showing no evidence for a disruption or a secondary instability even for aspect ratios as high as 80:1. The global evolution generally follows the standard SweetParker 2D reconnection model except for an accelerated reconnection rate at a very thin current sheet, due to the tearing instability and the formation of magnetic islands. An interesting conclusion is that despite the formation of fully $3 \mathrm{D}$ structures at small scales, the system remains close to 2D at global scales. We discuss the implications of our results for observations of the solar corona.
\end{abstract}

Subject Headings: Sun: corona - Sun: magnetic fields - Sun: reconnection 


\section{INTRODUCTION}

Magnetic reconnection has long been postulated to be the fundamental process underlying most solar activity (Parker 1972, 1988; Priest 1981; Priest \& Forbes, 2000). The basic scenario is that free energy in the form of electric currents is generated in the corona as a result of either the emergence of non-potential flux from below or the stressing of pre-existing coronal field by photospheric motions. Given the high Lundquist numbers $\left(\mathrm{S}>10^{10}\right)$ for typical coronal parameters, these currents could persist almost indefinitely, but some process (or processes) brings the scale of the currents down to values where the frozen-in-flux condition can be broken. The magnetic free energy then is released to the coronal plasma by reconnection. Depending upon the properties of the reconnection, the energy release may result primarily in heating, as in models for coronal heating (e.g., Klimchuk 2006; Rappazzo et al. 2008), or in violent mass motions, as in models for chromospheric explosions, coronal jets, and coronal mass ejections (Lynch et al. 2008, 2009; Pariat et al. 2009), or even in bursts of particle acceleration, as in flare models (e.g., Miller et al. 1997; Drake et al. 2006).

It is evident from this discussion that the formation of current singularities, which we shall generically term "current sheets," is central to reconnection and to solar activity. Various mechanisms have been discussed for current-sheet formation, but the one that we feel is most intuitive and most likely to apply to the corona is the null-point deformation process described in Syrovatskii's seminal work (Syrovatskii 1971, 1978a, 1978b, 1981). The fundamental argument is that for a magnetic topology with discontinuous connectivity - in particular, at a classic 2D X-type null point (actually an X line) with 
four topologically distinct flux systems - any stress applied to the system is likely to lead to discontinuous stress at the null and separatrices and, hence, to the rapid formation of current sheets there. This picture has been shown to be robust by many investigations, both analytic and numerical (Syrovatskii 1981; Antiochos 1990; Karpen et al. 1995, 1996, 1998; Birn et al. 1998; Antiochos et al. 2002). Although Syrovatskii formulated the theory originally for a $2 \mathrm{D}$ null-point configuration, the mechanism appears to be physically valid in 3D as well, with only straightforward modifications (Antiochos 1996; Edmondson et al. 2009, 2010).

In addition to the large-scale current-sheet formation process, the mechanism that breaks the frozen-in flux condition locally is critical for magnetic reconnection. In fact, it may well be that this microscale mechanism controls the energy release rate of the reconnection, thereby determining its global manifestations. After decades of intense study, a generally accepted picture has emerged for the simplest possible situation: steady-state reconnection in a 2D X-point topology under the MHD approximation. The studies have shown that if the dissipation mechanism, usually a simple isotropic resistivity, is spatially localized at the X point (Parker 1973; Biskamp 1993), then the reconnection takes on the Petschek form with a pair of slow-mode shocks, a current sheet short (in the 2D plane) compared to the global scale, and an inflow speed of the order of the Alfvén speed (Petschek 1964). On the other hand, if the resistivity is roughly uniform, then the reconnection adopts the Sweet-Parker (Sweet 1958; Parker 1963) form, with a current-sheet length of the order of the global scale and an inflow speed much less than the Alfvén speed. The physical origin of this sensitivity to the spatial localization of the resistivity is well understood, discussed originally by Parker (1972) and elaborated 
upon more recently by Kulsrud (2001). It should be emphasized, however, that both the Sweet-Parker and Petschek models are steady-state models only, and therefore do not address the problem of self-consistent current-sheet formation and disruption.

In previous MHD studies of solar activity in a 2.5D null-point coronal topology driven by photospheric motions, it was found that the resulting evolution is well represented by a hybrid of the Syrovatskii and Sweet-Parker models (Karpen et al. 1995, 1996, 1998). A smooth horizontal photospheric flow produced discontinuous stress at the null, resulting in its deformation to a current sheet and, hence, to reconnection there. Those studies all used numerical resistivity, which has no pre-defined spatial localization along the current sheet and, consequently, tends to produce a long global-scale current sheet and reconnection resembling the Sweet-Parker model rather than Petschek. A major new feature of these driven models, however, is that if the current sheet becomes sufficiently long, it is subject to tearing-mode instabilities (Furth et al. 1963; Bateman 1978), which result in the formation of magnetic islands and can enhance the inflow speed and reconnection rate (Karpen et al. 1995, 1996, 1998; Antiochos et al. 2002). The general conclusion, therefore, from the 2.5D MHD studies with numerical or uniform resistivity is that reconnection in the solar corona is well described by the standard Syrovatskii-Sweet-Parker scenario, but with the addition of magnetic island formation and the resulting dynamics.

A key question is whether this conclusion remains valid for a realistic 3D system (see Antiochos \& Dahlburg, 1997). Due to the intrinsic complexity of 3D topologies, current-sheet formation and reconnection in such systems have begun to be explored only recently (e.g., Lynch et al. 2008, 2009; Pariat et al. 2009; Edmondson et al. 2009, 2010). 
For systems that are fully 3D, such as an isolated X-point topology with no special symmetry (e.g., Pariat et al. 2009), the geometry is so different from the 2D model that it is difficult to relate the knowledge gained from the lower-dimensionality studies to the 3D evolution. One approach to bridging the gap between 2D and 3D is to consider a system that is initially $2 \mathrm{D}$, but to allow for a fully $3 \mathrm{D}$ evolution. The goal here is to be able to use some of our physical intuition gained from the large body of 2D reconnection studies in order to understand the more complex 3D system.

Such an approach has been used to investigate the 3D evolution of a pre-existing 1D current sheet (Dahlburg et al. 2003, 2005, 2006). Those studies produced an interesting and potentially important new result. As expected, the sheet tears to form 2D magnetic islands, but these are susceptible to an ideal 3D secondary instability, which grows much faster than the $2 \mathrm{D}$ tearing mode. The physical interpretation is that a magnetic island in 3D is actually a twisted flux rope, which can be susceptible to kinktype ideal instabilities that induce a burst of energy release. The results provide a physical mechanism for producing the type of reconnection bursts required by Parker's nanoflare model (Parker 1972) for coronal heating (Klimchuk 2006). Although they are interesting and potentially highly important, those results are not fully self-consistent, because the current sheet is assumed a priori rather than being generated by photospheric motions. Furthermore, the initial current sheet is $1 \mathrm{D}$ and of infinite extent (in principle) in the transverse directions, rather than having physical dimensions determined by the global scales in the problem.

In this paper we perform an investigation of the self-consistent formation and reconnection of a $3 \mathrm{D}$ current sheet driven by photospheric motions. The goal is to use as 
much as possible the insights gained from the $2 \mathrm{D}$ studies to understand the $3 \mathrm{D}$ evolution. For the initial topology we assume a 2D X line similar to previous studies (Karpen et al. 1995, 1996, 1998; Antiochos et al. 2002), except that we use Parker's (1972) ansatz of considering the magnetic field in a Cartesian box with the bottom and top of the box representing photospheric regions of different polarity. As will be evident below, this assumption allows us to stress the field with a simple 1D flow at the photosphere, which preserves the photospheric flux distribution there. Although the initial field is $2 \mathrm{D}$ and the driving flow is $1 \mathrm{D}$, the calculated evolution is fully $3 \mathrm{D}$ from the outset, allowing us to investigate the self-consistent formation and disruption of a 3D current sheet. The details of our numerical model and results are described in $\S \S 2$ and 3 below, and we draw conclusions about their implications for understanding coronal evolution in $\S 4$.

\section{NUMERICAL MODEL}

The magnetic field for our study is initially translationally symmetric and potential, i.e. current-free. We begin with an infinite line dipole of magnetic charge embedded in a uniform background field. The $x$ component of the vector potential for this field is given by

$$
A_{x 0}(\vec{x})=B_{0}\left[y-\frac{2 \alpha y}{y^{2}+z^{2}}\right] .
$$

The two terms on the right-hand side represent respectively a uniform background field of strength $B_{0}$ oriented parallel to the positive $z$ direction, and the field due to a single line dipole of strength $\alpha B_{0}$ (where $\alpha>0$ is a dimensionless scale factor) positioned at $(y, z)=$ $(0,0)$ and oriented anti-parallel to the background field. The $x$ direction is oriented along 
the line dipole, and the $y$ direction is the transverse horizontal coordinate. The general topology is that of a four-flux system with an X-type null point in the $y z$ plane (see Fig. 1). The separate flux systems are comprised of field lines that close from within the line dipole to the lower $z$ boundary to either side in $y$ or, alternatively, that connect the upper and lower $z$ boundaries to each other.

Figure 1 also indicates schematically the footpoint motion that is imposed at the top of our Cartesian box. To allow for an arbitrary horizontal displacement of the footpoints at the top $(z)$ boundary while simultaneously avoiding the complexity of inflow and outflow conditions at the side $(y)$ boundaries, we augmented the magnetic field above to make it periodic along the $y$ direction. An infinite series $(N \rightarrow \infty)$ of pairs of additional line dipoles positioned at $z=0$ and $y= \pm n d$ for $n=1,2, \ldots, N$, where $d$ is a prescribed periodicity length, contributes the following to the vector potential:

$$
A_{x 1}(\vec{x})=\alpha B_{0} y\left(\frac{2}{d}\right)^{2} \sum_{n=1}^{N}\left(\frac{1}{n^{2}}\right) \frac{\left(1-\frac{1}{n^{2}} \frac{y^{2}+z^{2}}{d^{2}}\right)}{\left(1-\frac{1}{n^{2}} \frac{y^{2}+z^{2}}{d^{2}}\right)^{2}+\left(\frac{1}{n} \frac{2 z}{d}\right)^{2}} .
$$

Although the sum is strictly periodic only as $N \rightarrow \infty$, we find in practice that $N=25$ terms are numerically sufficient on our domain. The resultant initial magnetic configuration is shown in Figure 1 over the periodic $y$ interval $[-d / 2,+d / 2]$ and the vertical $z$ interval $[h, h+d / 4]$, where we have chosen $h=d / 40$. For completeness, the convergence of the infinite series is demonstrated in the Appendix.

There remains the specification of the $x$ extent of our domain. Again, to minimize any influence of the boundaries, we elected to impose periodic conditions along the initially translationally symmetric $x$ coordinate. Anticipating that $y$ displacements of the 
order of the height of the domain, $\Delta z=d / 4$, would be imposed and introduce structure on that or smaller scales, we chose the $x$ domain extent to be $|x| \leq d / 4$.

We calculated the evolution of the field in Figure 1 with the Adaptively Refined Magnetohydrodynamics Solver (ARMS) (Welsch et al. 2005; DeVore \& Antiochos 2008; Lynch et al. 2008, 2009; Pariat et al 2009; Edmondson et al. 2009, 2010). We solved the following set of 3D compressible, ideal MHD equations:

$$
\begin{gathered}
\frac{\partial \rho}{\partial t}+\nabla \cdot \rho \vec{v}=0 \\
\frac{\partial \rho \vec{v}}{\partial t}+\nabla \cdot \rho \vec{v} \vec{v}+\nabla P=\frac{1}{4 \pi}(\nabla \times \vec{B}) \times \vec{B} \\
\frac{\partial T}{\partial t}+\nabla \cdot T \vec{v}+(\gamma-2) T \nabla \cdot \vec{v}=0 \\
\frac{\partial \vec{B}}{\partial t}=\nabla \times(\vec{v} \times \vec{B})
\end{gathered}
$$

where all variables have their usual meanings. In the adiabatic equation for the temperature, the ratio of specific heats is $\gamma=5 / 3$. We have used the ideal gas law $P=\rho R T$ for the plasma equation of state, where $R$ is the gas constant. Gravity is not included in this calculation, since we are interested in the local structure and evolution of current sheets in the high-temperature corona.

The MHD equations above can be made dimensionless by extracting scale factors for the mass density $\rho_{s}$, magnetic field strength $B_{s}$, and characteristic length $L_{s}$. The characteristic length $L_{s}$, for example, can be set to that of a coronal structure of interest; the associated normalized length in our simulation is $h$, the depth of the line dipole. The mass density $\rho_{s}$ and field strength $B_{s}$ are similarly free parameters; their normalized counterparts $\rho_{0}$ and $B_{0}$ will be specified below. As a result of this scaling, all values of the MHD variables in the simulation are of order unity. For example, the initially 
uniform mass density $\rho_{0}$ and background field strength $B_{0}$ are chosen to set the global average Alfvén speed $V_{A}$ (defined below) to unity. We set the relative line-dipole strength $\alpha$ so that the null is positioned well inside the simulation domain, insulating the currentsheet formation and dynamics from any uncontrolled boundary influences. The initially uniform thermal pressure $p_{0}$ is chosen to assure that the low-beta condition (thermal pressure much smaller than magnetic pressure, so that the dynamics are magnetically dominated) holds everywhere in the domain other than in the immediate vicinity of the null line. Figure 2 illustrates the plasma beta across the inner portion of the magnetic structure, the white contour showing the $\beta=1$ surface. Only within this small region in the neighborhood of the null line does the plasma beta rise substantially above unity. We emphasize, however, that although the system as a whole is low-beta, the thermal pressure nevertheless plays an important role in the evolution. It dominates in the small region near the null line, so that the dynamics of current-sheet formation and subsequent reconnection are critically dependent upon the thermal pressure being finite rather than zero.

In the low-beta limit, driving motions that are slow compared to the Alfvén speed yield an approximately force-free evolution. This regime is appropriate for current-sheet formation in the corona in response to slow photospheric driving. Much like the plasma beta, the Alfvén speed varies most strongly over a small region in the vicinity of the null line, where it approaches zero. Elsewhere, the Alfvén speed varies smoothly, approaching a uniform value well away from the line dipole and its periodic images. It is convenient to define the global average Alfvén speed $\left\langle V_{A}\right\rangle=\sqrt{2 E_{M} / M}$, where $E_{M}$ is the total magnetic energy and $M$ the total mass in the system. The scale factors for mass density 
$\left(\rho_{s}\right)$ and magnetic field $\left(B_{s}\right)$ can be chosen such that $\left\langle V_{A}\right\rangle=1$ in our initial setup in

Figure 1. As a consequence, all velocities in our simulation will be measured in units of the initial global average Alfvén speed $\left\langle V_{A}\right\rangle$ of the corresponding coronal configuration. We noted earlier that the length scale factor $L_{s}$ fixes the scaled line-dipole depth $h$, which defines a global average Alfvén time $\left\langle t_{A}\right\rangle=h /\left\langle V_{A}\right\rangle$. We can set $h=1$, whence $\left.<t_{A}\right\rangle=1$. As a result, the elapsed time in our simulation similarly will be measured in units of the initial global average Alfvén time $<t_{A}>$ in the corona.

We energize the system by imposing a sub-Alfvénic, spatially uniform flow all across the upper $z$ boundary, as indicated schematically in Figure 1. In order to minimize transient effects as the motions get underway, we employ a gradually accelerating cosine profile in time:

$$
\begin{aligned}
& \vec{V}=V_{y}(t) \hat{y} \\
& V_{y}(t)=\left\{\begin{array}{cl}
\frac{V_{0}}{2}\left[1-\cos \left(\pi \frac{t}{t_{0}}\right)\right] & 0 \leq t \leq t_{0} \\
V_{0} & t>t_{0}
\end{array}\right.
\end{aligned}
$$

The magnitude of the imposed flow is $V_{0} \ll<1$, slow compared to the global average Alfvén speed, while the duration of the smooth acceleration phase is $t_{0}>>1$, long compared to the global average Alfvén time. An accumulated displacement $V_{0} t_{0} / 2 \sim h$ distorts the null line sufficiently to create a substantial current sheet by the end of the acceleration phase. After time $t=t_{0}$, the driver speed is held constant, so that any subsequent burstiness in the system will be an intrinsic property of the dynamics at the sheet rather than a response to a time-variable driver. Line-tied conditions are imposed at 
both $z$ coordinate boundaries, with the footpoints held fixed (zero flow speed) at the lower boundary. Consequently, the only way that the magnetic field can change topology is through reconnection.

Figure 3 shows the initial numerical grid for the simulation. Our base grid consisted of $2 \times 2 \times 1$ blocks distributed uniformly in $\{x, y, z\}$, with $8^{3}$ grid points per block (note that only every other grid point is shown in the figure). We then imposed an initial refinement to a maximum of 6 levels, over a subvolume that we anticipated would be sufficient to encompass fully the developing current sheet. This region, which has the highest density of grid lines (cyan) in Figure 3, is centered rightward and upward from the null line. Beyond this subvolume, the grid coarsens by a factor of two at each change in refinement. We employed adaptive mesh refinement (AMR) to increase further the grid resolution near the strengthening current sheet as the simulation progressed. Our mesh-refinement criterion tests the gradient of the magnetic-field magnitude in regions of weak to moderate field strength. The grid was allowed to refine up to a maximum level of 9, corresponding to a finest uniform grid of $4096 \times 4096 \times 2048$ cells if employed over the entire domain. The resultant minimum grid spacing, $\delta=2.44 \times 10^{-4} d$, is far smaller than the scale of the initial magnetic structures.

\section{RESULTS}

\subsection{Initial Configuration and Current Sheet Development}

The domain size is set to $d=40 h=40$, so that the simulation was performed on the space $\{(x, y, z) \mid x \in[-10,10], y \in[-20,20], z \in[1,11]\}$. We chose a relative line-dipole strength $\alpha=20 / 3$, which places the initial null line near domain center in the $z$ direction 
(see Figure 1). The scale factor $B_{s}$ for the absolute field strength is chosen so that the simulation background field $B_{0}=3$. We found that the root mean square field magnitude in our initial configuration is approximately $\sqrt{4 \pi}$. Choosing $\rho_{0}=1$ then yields the global average Alfvén speed $\left\langle V_{A}\right\rangle=1$, as desired. We set the thermal pressure $p_{0}=0.01$. The global average plasma beta therefore is $\langle\beta\rangle=0.02$, while the minimum value $\beta_{\min } \sim 10^{-4}$ is attained at the bottom $z$ boundary, in the closed field of the line dipole (see Figure 2).

For the imposed flows at the top $z$ boundary, we chose a peak speed $V_{0}=0.045$ and a corresponding duration for the acceleration phase $t_{0}=100$. The resultant time profile of the boundary flow speed is shown in Figure 4 . At times $t \geq t_{0}=100$, the flow is held steady at its peak value. Since the plasma and field are fixed at the bottom $z$ boundary, we can expect the average velocity over the entire volume to approach roughly $V_{0} / 2$. For the assumed values of $\rho_{0}, V_{0}$, and system size $d$, the predicted asymptotic kinetic energy integral over the volume is $E_{K}=2$. The simulation closely adheres to this expectation, as shown by the time profile of kinetic energy in the top panel of Figure 5.

In the early development stage, $t \leq 40.19$, the driving speed and footpoint displacements ramp up slowly and only weak currents appear in the corona. If the line dipole were not present, so that the initial field was uniform and vertical everywhere, then the field would remain nearly potential throughout the evolution. The photospheric motions would generate a uniform $B_{y}$ that increases linearly with the total photospheric displacement in the $y$ direction, and the field lines simply would tilt increasingly away from the vertical as their top footpoints suffer ever greater displacements. The closed bipole flux, however, acts like an obstacle to the background field, and the field must deform around the bipole flux as it tilts. The field strength is small in the neighborhood of 
the null, so that field lines passing through that region cannot sustain large stresses. Those above the null connect to the top $z$ boundary, and the imposed flows displace them laterally with relative ease. In contrast, those below the null connect to the bottom $z$ boundary, where the field magnitude is quite large and the footpoints are fixed. Inside the bipole near the line source, strong volumetric currents appear as the field resists the stresses transmitted by the motions imposed at the top boundary.

The process of current-sheet formation is intuitively simple. Prior to onset of the boundary driving motions, inner and outer spine lines (actually planes in 3D) depart the null point toward the bottom and top $z$ boundaries, respectively, and are perfectly vertical. The imposed driving motion moves the top footpoint of the outer spine to the right, which imparts a stress to the field and drags the lower end of the outer spine to the right. The inner spine, however, feels no stress and stays more or less fixed. Consequently, the spines dislocate, causing the null point to deform. This process begins as soon as the motions get underway, initiating the development of the current sheet. The small but finite gas pressure limits the narrowness of the current sheet at the outset. Figure 6 shows the system configuration at the initial and final times, $\mathrm{t}=0.0$ and 147.64 seconds, (the corresponding movie is available in the electronic version of the paper). The field lines are shown in white against the perpendicular current contour background (the strong current, $\left|J_{x}\right| \geq 5$, region is cyan). The singular current sheet is clearly seen between the dislocated spine lines in the left figure.

As the driving flows accelerate, the null line stresses further, forming an increasingly strong neutral sheet. The adaptive mesh refinement first turns on at time $t=$ 40.19 (see Figure 5), as the null deforms substantially and its current structure steepens to 
approach the grid scale at the initial maximum refinement level (6). At the successive times $t=40.19,50.317$, and 60.187 , the maximum refinement level increases to 7,8 , and finally 9 , as the grid subdivides to resolve the continually thinning sheet. The ongoing boundary motions further lengthen the current sheet and increase the volume occupied by grids at the highest refinement level (9), as illustrated at time $t=70.003$. At each of these discrete times, as well as at other intervening times in the simulation, there are strong changes in the slope of the kinetic energy plot. These evidently are associated with the internal restructuring of the current sheet as the grid refines in discrete jumps. Through the remainder of the acceleration phase and during the subsequent phase of constant imposed boundary motions, at times $t \geq 70.003$, the grid has reached its maximum refinement level (9) over the entire sheet between the inner and outer spine lines. At this level of refinement, the current sheet half-thickness $a$ is approximately the finest grid spacing, $\delta \approx 2.5 \times 10^{-4} d=1.0 \times 10^{-2}$.

At times $t \leq 70$, the current sheet lengthens and intensifies as the driving flows accelerate. Subsequently, the lengthening subsides as the imposed flows reach top speed. At times $t \geq 100$, the driving flows are constant. During this late phase, the current sheet exhibits a more or less steady state of approximately fixed length (see Figure 7), in which plasmoids repeatedly form within the sheet and are ejected out of one end or the other, as described in detail below. In order to interpret our findings using results from MHD reconnection theory, it is necessary to estimate the effective magnetic diffusivity $\eta$ in the simulation. Balancing the inflow speed $V_{i}$ of magnetic flux into the current sheet against the resistive annihilation across the sheet yields

$$
\frac{V_{i}}{a}=\frac{\eta}{a^{2}}
$$


whence

$$
\eta=V_{i} a .
$$

Combining this estimate with a characteristic global scale $L$, such as the half-length of the current sheet, and the local Alfvén speed $V_{A}$, the effective Lundquist number $S$ then is

$$
S=\frac{V_{A} L}{\eta}=\frac{V_{A} L}{V_{i} a} .
$$

Averaged along the current sheet for times $t \geq 100$, we find that the current-sheet inflow and Alfvén speeds are approximately $V_{i}=0.03$ and $V_{A}=0.45$, respectively. The spine-tospine separation length is $2 L=0.04 d=1.6$, and as noted above the half-thickness of the sheet is $a=0.01$. Thus, the effective numerical resistivity and Lundquist number for the fully developed, high-resolution current sheet can be estimated to be $\eta=3 \times 10^{-4}$ and $S=$ $1.2 \times 10^{3}$, respectively, during the approximately steady-state phase of the evolution. It should be emphasized, however, that these values for $\eta$ and $S$ apply only at the current sheet, itself. Outside the current sheet, the magnetic structure is very well resolved and the evolution is effectively ideal.

\subsection{Plasmoid Formation and Ejection}

The relatively long-wavelength resistive tearing mode is the most important MHD instability in the developing current sheet. In 2D, resistive tearing of the sheet generates alternating X-and O-type field topologies all along its length (Biskamp 1993). Flux accumulating around the $\mathrm{O}$ points creates magnetic islands of locally closed field lines,

which trap the reconnection-jet plasma and thus form enhanced-density plasmoids. In 3D, the additional degree of freedom enables the development of much richer topologies. In 
the absence of a guide field, as in our case, 3D perturbations with variations along the nominal symmetry axis generate quasi-ergodic, slinky-like field lines via reconnections between neighboring cross-sectional planes. Current sheets of finite length, whether 2D or 3D, eject their fully developed magnetic islands/plasmoids in the direction of the closest terminus of the sheet. Therefore, we differentiate the tearing-mode process into two stages: plasmoid formation and plasmoid ejection. The formation stage consists of the initial field line pinching and linear growth of the plasmoid; its characteristic length scale is the current sheet half-thickness (i.e., $a=0.01$ ). The ejection stage occurs once the plasmoid has grown to the order of the current-sheet thickness, so that nonlinear effects come to dominate the dynamics. We discuss the final scale of the ejected plasmoids in the following section.

The linear growth time $\tau$ of the tearing mode is intermediate between the short Alfvén time $\tau_{A}=a / V_{A}$ and the long resistive time $\tau_{\eta}=a^{2} / \eta$ of the sheet (Furth et al.1963). It depends upon the large-scale properties of the current-sheet magnetic field, the sheet thickness, the wavelength along the sheet, and a weighted geometric average of the two characteristic time scales. The linear growth rate can be expressed as (e.g. Bateman 1978)

$$
\gamma \approx 0.9 \frac{(k a)^{2 / 5}\left(\Delta^{\prime} a\right)^{4 / 5}}{\tau_{A}^{2 / 5} \tau_{\eta}^{3 / 5}}
$$

where $\Delta^{\prime}$ is the jump in the logarithmic gradient of the magnetic-field perturbation across the current sheet and $k$ is the wavenumber along the sheet. The quantity $\Delta^{\prime}$ depends solely on the properties of the ideally evolving field, and at moderately long wavelengths $k a<1$ can be estimated as (Furth et al. 1963)

$$
\Delta^{\prime} a \approx \frac{2}{k a} .
$$


The tearing-mode growth rate then becomes

$$
\gamma \approx \frac{1.6}{(k a)^{2 / 5} \tau_{A}^{2 / 5} \tau_{\eta}^{3 / 5}},
$$

which can be inverted to give the growth time

$$
\tau \approx 0.6(k a)^{2 / 5} \tau_{A}^{2 / 5} \tau_{\eta}^{3 / 5}
$$

previously used to interpret tearing and island formation in a strictly $2 \mathrm{D}$ current sheet (e.g. Karpen et al. 1995).

Throughout the evolution of our 3D system, the discernible tearing wavelengths are between one-fourth and one-half of the total length. For a current-sheet aspect ratio $L / a=80$ and at most two wavelengths along the sheet half-length $L$, we find

$$
\tau \leq 0.3 \tau_{A}^{2 / 5} \tau_{\eta}^{3 / 5}
$$

Recalling that the current-sheet Alfvén speed $V_{A}=0.45$ and effective magnetic diffusivity $\eta=3 \times 10^{-4}$, we find $\tau_{A}=0.02$ and $\tau_{\eta}=0.3$. The linear growth time during the plasmoid formation stage then is $\tau \leq 0.03$. This is much shorter than the characteristic inflow time for the sheet, $\tau_{i}=a / V_{i}=\tau_{n}=0.3$, so that applying the linear perturbation analysis for an equilibrium sheet to our simulation is reasonable. In addition, many e-folding times elapse during the late sheet-lengthening and steady-state phases of the evolution. This provides ample opportunities for new islands to be seeded at small amplitude, grow to discernible size, and saturate in the nonlinear regime. For example, the top panels of Figure 8 (at times $t=111.66,112.02$, and 112.54) illustrate emergence from the noise of a sheet-spanning plasmoid over some thirty linear growth times.

For the most part, we find that the plasmoids initially form near the center of the current sheet. Since the typical tearing wavelength is one-fourth to one-half of the sheet 
length, we find on average that there are two to four irregularly shaped plasmoids in any given perpendicular $y z$ plane. The plasmoids generally are well separated from one another along the sheet within the $y z$ plane. Nonlinear effects saturate the rapid growth of the plasmoid when its width becomes comparable to that of the current sheet: at that point, the locally available free energy of the magnetic field is exhausted and a new quasi-equilibrium state is attained. Thereafter, the plasmoid evolution is governed by the gradually evolving state of the current sheet as a whole.

The ejection time for individual plasmoids is somewhat variable during the evolution, depending upon the precise location of formation along the current sheet and the instantaneous length of the sheet itself. The ejection speed reflects the local outflow velocity of the reconnection jets within the sheet, which is approximately the Alfvén speed $V_{A}=0.45$. Plasmoids sweeping out the ends of the sheet - especially the larger plasmoids - split the sheet there, temporarily reducing its length. A characteristic time scale $\tau_{e}$ for plasmoid ejection is the Alfvén time across the maximum sheet half-length $L$ : $\tau_{e}=L / V_{A}=1.8$. Figure 8 illustrates a typical plasmoid ejection in the panels at times $t$ between 113.08 and 114.95 , during which a plasmoid that formed roughly in the center of the sheet is ejected from it over the prescribed Alfvénic ejection time scale $\tau_{e}$ (the corresponding movie is available in the electronic version of the paper).

Our simulation is fully 3D at all times, and there is no guide field imposed along the initially symmetric axis of the magnetic configuration. Consequently, the tearing-mode perturbations that seed the formation of the magnetic islands and plasmoids in each $y z$ plane do not necessarily develop coherently over any significant length along $x$ between the planes. In this simulation of a truly neutral sheet, the only guide-field component that 
emerges is seeded by 3D numerical perturbations. This limits the correlation length along the nominal symmetry axis of the current sheet to only a few grid cells.

As a consequence of this lack of correlation along the third dimension, together with the short time for island growth relative to the long time for sheet development, the plasmoids that form are short and evolve effectively independently on different $y z$ planes. Although there is some evidence for kinking of these short structures, they do not become sufficiently large to affect the overall integrity of the sheet. We illustrate this point in Figure 8 (and the corresponding movie available in the electronic version of the paper), which shows the current sheet in the $x y$ plane as viewed from above. The times are the same as those in Figure 8, and the white stripes mark the $y z$ plane shown in cross-section in the previous figure. Purple shading shows strong current-density magnitude $(|J| \geq 5)$, while red shading highlights the plasmoid density enhancements $(\rho \geq 2)$. The white arrows in Figure 8 track the plasmoid formation, evolution, and ejection indicated by the black arrows in Figure 8. The 3D plasmoids evolve quasi-independently along the third dimension, at some times separating and at other times coalescing as they interact during their transit along the sheet. Although the resultant structures occasionally kink, they never extend very far along the third dimension and are ejected quickly enough that the current sheet always maintains its overall stability. It is obvious from Figure 8 that the plasmoid structure is highly irregular and fully $3 \mathrm{D}$ at all times.

\subsection{Quasi-Steady Reconnection Dynamics}

The late-phase, approximately steady state of our system $(t \geq 100)$ consists of a

dynamically stable, 3D neutral current sheet with an aspect ratio of around 80:1. Clearly, 
the system dynamics favor a rather quiet steady-state reconnection with occasional formation and ejection of localized magnetic islands, rather than an explosive or bursty reconnection that results in large-scale turbulent breakup of the current sheet. These dynamics are a direct consequence of the nature of the driving flows, which produce magnetic stresses localized primarily to the separatrices between flux systems. These stresses relax directly via the observed reconnection. To that end, the fully developed current sheet attains a more or less constant length between the displaced spine lines, although the instantaneous length in any particular $y z$ plane fluctuates somewhat with each plasmoid ejection. This implies that the field-stressing and reconnection rates have converged to a common value, at least in a time-averaged sense.

Figure 10 shows the color-shaded velocity magnitude at the $x=0$ mid-plane for several times $t=100.1,111.66,115.74$, and 147.64 , typical of the phase of statistically steady dynamics. The high-speed $(|V| \geq 0.2)$ reconnection jets are clearly seen as the purple-shaded regions within and at the ends of the current sheet. White arrows show the vector velocity direction, essentially perpendicular to the sheet at either side of it, and principally along the sheet within it. Black contours illustrate the extent of the strong currents $(|J| \geq 5)$ in the system. Both the average inflow speed $V_{i}$ across the sheet and average outflow jet speed $V_{o}$ along the sheet increase gradually with time. In addition, the flow speeds below the current sheet are slightly lower while the Alfvén speeds are slightly higher, compared to those above the sheet. This reflects the higher field strength within the closed line-bipole region, which is more resistant to stressing. Table 1 enumerates the average inflow and outflow speeds and their ratios, at the times shown in Figure 10. We note that there also is a slight rising trend versus time of the reconnection 
rate, as measured by the inflow/outflow ratio. Evidently, the sheet has not yet attained a perfectly balanced steady state, as it continues to adjust to the fixed driving motions and the intermittent dynamics of plasmoid formation and ejection.

It is instructive to compare our results with those predicted for steady-state SweetParker and Petschek reconnection (e.g. Kulsrud 2005). The values in Table 1 show that the average ratio of inflow to outflow speeds over the interval is $V_{i} / V_{o}=0.11$, whereas the ratio of current-sheet thickness to length is $a / L=0.012$. These numbers are very different from each other and from the common value predicted by Sweet and Parker, $1 / \sqrt{ } S=0.03$, for our Lundquist number $S=1.2 \times 10^{3}$. Compared to Sweet-Parker, our quasi-steady current sheet is thinner yet its inflows are faster, by factors of about three. In steady-state Petschek theory, on the other hand, the reconnection region along the current sheet shrinks to an effective half-length $L^{\prime}$ given by

$$
\frac{L^{\prime}}{L}=\left(\frac{V_{o}}{V_{i}}\right)^{2} \frac{1}{S},
$$

while the inflow/outflow ratio satisfies

$$
\frac{V_{i}}{V_{o}}=\frac{\pi / 8}{\ln \left[\left(V_{i} / V_{o}\right)^{3} S\right]}
$$

For our Lundquist number $\mathrm{S}$, the predicted inflow/outflow ratio $V_{i} / V_{o}=0.19$, and the reconnection region shortens to $L^{\prime}=0.02$ so that $a / L^{\prime}=0.5$. The latter is commensurate with the size of our magnetic islands; however, the Petschek inflow speed and associated reconnection rate are too high by nearly a factor of two. Thus, our dynamically tearing current sheet exhibits an overall Sweet-Parker-like structure with locally reconnecting regions at the Petschek scale, but with an inflow/outflow ratio and reconnection rate that are intermediate between these $2 \mathrm{D}$, steady-state reconnection models. 
The key point is that our driven current sheet is physically different than that of the Sweet-Parker or Petschek reconnection models. In these steady-state models the reconnection rate, (the inflow velocity), is the important derived parameter; but, for our current sheet, the inflow/outflow ratio is fixed by the initial and boundary conditions. The current sheet forms due to the horizontal displacement (at imposed speed $V_{0}$ ) of the initially uniform vertical field (at strength $B_{0}$ and mass density $\rho_{0}$ ). Thus, the inflow speed into the sheet is $V_{i} \approx V_{0}=0.045$ and the outflow speed within the sheet is the local Alfvén speed, $V_{o}=V_{A} \approx B_{0} / 2 \sqrt{ } 4 \pi \rho_{0}=0.42$, using $B_{0} / 2$ as an average field strength within the null region. Their ratio $V_{i} / V_{o}=0.11$ is fully prescribed by these parameter choices; hence, the question of the reconnection rate is not relevant to our system.

The important question that does remain, however, is the scaling of our current sheet scale with increasing $S$, which is equivalent to increasing grid refinement. There are four critical parameters that describe a general reconnecting current sheet. The first and most important is the current sheet width, $a$, or more exactly, the scale at which the frozen-in flux condition is broken and the reconnection of field lines occurs. For our calculations, which use numerical resistivity, the flux-breaking scale is simply the grid size. Although this choice may seem arbitrary, it is actually the most appropriate resistivity model for an MHD calculation. The flux breaking in the Sun's corona and in most astrophysical plasmas is expected to occur at kinetic scales such as the ion skin depth or gyroradius; therefore, no MHD model can capture the physics of the reconnection region rigorously. All the kinetic studies have shown, however, that the reconnection sets in at some fixed scale, such as the ion skin depth or gyroradius, (e.g., Cassak et al 2005; Yamada 2007). Therefore, the best that can be done in an MHD 
calculation is to set the diffusion to occur at as small a fixed scale as possible. Our ARMS code does exactly this by adding diffusion to the ideal MHD solution whenever the current structure gets down to the grid scale.

The next key parameter in the problem is the length of the current sheet, $L$. In our system, this length is determined by the current sheet formation process and the scales of the initial magnetic structure. The scale of the high-beta region around the initial null point is of order the global scale. In order for this region to deform to a neutral sheet whose width decreases down to the grid scale, the region must suffer a significant deformation, at least, of order its initial size. This simple argument implies that the length of current sheets that form in the Sun's corona must be of order the global size of the relevant magnetic flux systems, as found in our simulation.

The final two parameters are the inflow and outflow velocities. The former is set directly by the imposed boundary flow. The latter is set by the Alfven speed inside the embedded bipole. Note that the current sheet forms, because the stress added to the external field causes it to press down against the field of the bipole. It does not require an enormous deformation of the bipolar field to decrease the current sheet width down to the grid scale; hence, the field in the inflow region just outside the current sheet is of order the initial bipole field. In other words, there is no significant flux pile-up in our system.

We conclude that the scale and magnitude of the global fields and imposed driving motions completely determine all the important parameters of the reconnecting current sheet. But this result seems impossible, because there are certain constraints that the system must satisfy, such as mass and flux conservation, which should impose additional, independent constraints on the current sheet parameters. The existence of 
these additional constraints is the reason for the appearance of magnetic islands (plasmoids). The plasmoids break up the global-scale current sheet into substructures that introduce new scales to the system; thereby, allowing the conservation constraints to be satisfied while the global current sheet parameters remain fixed by the input scales and driving.

Consider, in particular, mass (or flux) conservation. For the simplest possible current sheet structure, the Sweet-Parker case, this constraint requires that $V_{o} / V_{i}=L / a$; but we find that $V_{o} / V_{i}=9.1$; whereas, $L / a=83$. The critical point, however, is that the mass flux out of the global current sheet layer is carried not by the individual reconnection jets that have width, $a$, but by the expulsion of plasmoids. These have an average width, $w$, of order 10, which is sufficient to balance the mass flux into the global sheet. Note that this result implies that the plasmoid width, $w$, should be independent of Lundquist number, or equivalently, the grid scale of the simulation. Furthermore, the plasmoid width will scale directly with the driving velocity, $V_{i}$, assuming that the initial conditions of the simulation are unchanged.

Mass conservation also determines the average number of plasmoids in the global current-sheet layer. As the plasmoids separate, the current sheet between them grows to the maximum length compatible with mass conservation, $L_{M}=a V_{o} / V_{i}$, beyond which new plasmoids must appear. For fixed driving and Alfven speeds, this limit implies that the number of plasmoids scales with the current sheet width, $a$, which for our current sheet is proportional to the Lundquist number. On the other hand, the maximum size of the plasmoids is independent of Lundquist number and set only by the reconnection rate and the global current sheet length. We conclude, therefore, that driven current sheets in 
the Sun's corona will develop a distribution of plasmoids ranging in scale from formation at a scale of order $a V_{o} / V_{i}$, to expulsion at a scale of $L V_{i} / V_{o}$. This result is qualitatively similar to what is found for studies of current sheets initially in equilibrium, such as a Harris sheet (e.g., Loureiro et al. 2007; Bhattacharjee et al. 2009). The exact scaling of plasmoid number with S may be somewhat different depending on whether one is examining a driven or an unstable system, but all the studies appear to agree that the density of plasmoids increases as some power of Lundquist number.

We note finally that the fraying and splitting at the ends of the current sheet in response to plasmoid ejections create localized, transient Petschek-like geometries there. Figure 11 illustrates this effect by comparing the strong current magnitude and plasma density, together with overlying magnetic field lines. In the bottom panel, the field lines (white) clearly exhibit sharp Alfvénic bends where the current sheet splits at the right side. These Petschek-like structures at the ends of the sheet are very short and transient, developing as the current sheet splits when plasmoids are ejected and dissipating as a single sheet reforms via the subsequent relaxation of the field.

\section{Conclusion}

The calculations presented in this paper constitute, in a sense, a minimalist extension of $2 \mathrm{D}$ current-sheet formation and reconnection to the fully $3 \mathrm{D}$ regime. The advantages of our approach are that it lends itself to straightforward comparisons with previous $2 \mathrm{D}$ and 3D work and allows us to employ very high numerical resolution at the current sheet. The results have a number of important implications for solar activity and for magnetic reconnection more generally. 
First, it is clear that a current sheet forms due to the imposed photospheric motions, even though the motions are the smoothest possible uniform flow. This result demonstrates convincingly that a multipolar topology, even the extremely simple one of our model, inevitably will lead to current sheets in the corona. The formation process is physically identical to the Syrovatskii model: an initial X-type null deforms under the applied stress into a neutral current sheet bounded by two Y-type nulls. For our particular magnetic configuration and photospheric driving, the currents are confined to the deformed null-point region and are small at the separatrix surfaces. If we were to apply a flow at the upper surface that drives the field in the initially symmetric direction, parallel to the $x$ axis, however, current singularities would form along the separatrices as well.

The current-sheet formation process is rapid and efficient. It is evident from Figure 5 that the structure at the deformed null thins down to the maximum resolution for a fairly small displacement of the outer spine, less than $20^{\circ}$ of tilt from the vertical. We expect the thinning to occur exponentially (e.g. Antiochos et al. 1999), so that even for the very large Lundquist numbers of the real corona, current structures down to the dissipation scale form on essentially ideal time scales. An important implication of this result is that current sheets are likely to be present in the corona even though the magnetic field there is generally observed to be near the potential state. We see from Figure 5 that a modest distortion from the potential state is sufficient to generate structure at the dissipation scale. Note, however, that these current singularities occur at very specific locations: the topological boundaries between different flux systems. It remains an open question whether current sheets form ubiquitously throughout the corona (Parker 1972; van Ballegooijen 1985; Antiochos 1987). 
Once the current sheet lengthens sufficiently that reconnection can compete with the slow driving motions, the sheet stops growing. From this point on (Figure 7), the structure near the null is quasi-static, with the stretching by photospheric displacements balanced by the shrinkage due to reconnection. Of course, the system is not in a true steady state, because the flux in the right-hand arcade of Figure 7 decreases continuously due to the reconnection, while the flux in the left-hand arcade increases. Eventually the flux imbalance will reach the point at which the reconnection stops, but we see no evidence for this saturation, at least for the footpoint displacements in our simulation. Although not extreme, the displacements clearly are substantial. Figure 7 shows that by the end of the calculation, the footpoints on the upper boundary have moved a distance on the order of the size of the closed bipole. It is intriguing that in this quasi-steady state, the angle of the uniform background field with respect to the vertical is roughly $20^{\circ}$, close to the stress angle required by Parker for his coronal-heating model (Parker 1983). We emphasize, however, that the topology of our system is significantly different from that of Parker's.

A striking result of the simulation is the extreme stability of the current sheet and reconnection dynamics, as shown in Figures 6 and 9. The dynamics are much less explosive than our simulations of reconnection with isolated 3D null-point topologies (Pariat et al. 2009; Edmondson et al. 2009, 2010), and even less impulsive than the 2.5D simulations with null lines (Karpen et al. 1995, 1996, 1998). The origin of the differences between the results here and our previous simulations of coronal reconnection lies in the form of the driving. First, the photospheric motions in the present calculation deform only the large-scale external flux system, not the small-scale embedded bipole; 
consequently, there is no need for the free energy to spread outward from an isolated small region. Second, the motions inject no helicity into the corona; the field lines are not twisted or sheared. Therefore, reconnection, by itself, can lower the magnetic energy toward the potential-field minimum without having to propagate helicity over global scales. The implications for the corona are that slow photospheric driving of the largescale coronal field can result in quasi-steady energy release via reconnection, without any dramatic dynamics. Such a process is exactly what is required to account for the nearsteady heating of solar quiet regions.

The result that the current sheet is so stable is especially surprising, given that the sheet achieves aspect ratios as high as $80: 1$. The dynamics are generally consistent with steady-state Sweet-Parker reconnection except for the addition of magnetic-island formation. These plasmoids carry most of the mass and are ejected from one current sheet terminus or the other on the Alfvén time scale for the sheet length. We emphasize, however, that structure in the $x$ direction does form at both small and global scales, and that this 3D structure is critically important. The small-scale structure is due primarily to the formation of plasmoids at random locations along the initial symmetry axis of the sheet. The large-scale structure is evident as a periodic arrangement of the field lines with exactly four wavelengths across the domain (see Figure 12). Clearly, this structure is seeded by the finite grid of the calculation, as is to be expected, but only the small-scale features are physically significant.

We claim that the small-scale structure in the $x$ direction is directly responsible for the lack of burstiness in the reconnection. Figure 8 shows that with regard to the plasmoids, there is absolutely no symmetry in the $x$ direction. The plasmoids are fully $3 \mathrm{D}$ 
and form at random locations throughout the current sheet. This 3D structure suppresses the secondary instability that generated the bursts of reconnection seen in previous current-sheet studies (Dahlburg et al. 2003, 2005, 2006). In those simulations, the system was seeded with 2D perturbations that produced magnetic islands (actually twisted flux tubes, owing to the finite guide field) with infinite length in the $x$ direction. The secondary instability is basically an ideal, kink-like mode of the highly twisted flux tubes. Two-dimensional perturbations of a 3D system, however, are somewhat unphysical, because they assume that the noise has very long coherence length along a preferred direction. In the present simulation, the noise is fully $3 \mathrm{D}$ and leads to plasmoids whose coherence scale length in the $x$ direction is similar to that along the current-sheet length in the $y z$ plane. Hence, there is essentially no energy available to be released by a kinking instability. Furthermore, the 3D nature of the magnetic islands that we find is likely to limit the effectiveness of the islands in trapping particles, which could have important implications for flare acceleration mechanisms (e.g. Drake et al. 2006).

An important caveat to these conclusions is that there is no guide field in our simulation. Due to the form of the driving, a true neutral sheet is generated. It may well be that if a guide field were present it would introduce a much longer coherence scale length along the $x$ direction, so that much longer flux-tube-like magnetic islands would form. The situation is analogous to the issue of flare-ribbon formation. A strong guide field provides the reconnection coherence required to produce the distinctive ribbon structure. On the other hand, if the guide field is too strong, it will suppress any kinking via secondary instability (Dahlburg et al. 2003, 2005, 2006). We conclude, therefore, that 
simulations with varying amounts of guide field should be the next step in studying the formation and reconnection of coronal current sheets.

\section{Acknowledgements}

This work was supported in part by the NASA HTP and SR\&T programs. JKE acknowledges support from the NASA GSRP Program. The numerical simulation was performed under a grant of time from the DoD High Performance Computing Modernization Program.

\section{Appendix A}

To demonstrate that the vector-potential series

$$
s(y, z ; d)=\sum_{n=1}^{\infty}\left(\frac{1}{n^{2}}\right) \frac{\left(1-\frac{1}{n^{2}} \frac{y^{2}+z^{2}}{d^{2}}\right)}{\left(1-\frac{1}{n^{2}} \frac{y^{2}+z^{2}}{d^{2}}\right)^{2}+\left(\frac{1}{n} \frac{2 z}{d}\right)^{2}}
$$

converges, note that the last term in the denominator is positive definite and also that the quantity $\varepsilon^{2} \equiv\left(y^{2}+z^{2}\right) / d^{2}$ satisfies $0<\varepsilon^{2}<1$ on our simulation domain. Hence, 


$$
\begin{aligned}
s(y, z ; d) & <\sum_{n=1}^{\infty}\left(\frac{1}{n^{2}}\right) \frac{1}{\left(1-\frac{1}{n^{2}} \frac{y^{2}+z^{2}}{d^{2}}\right)} \\
& =\sum_{n=1}^{\infty} \frac{1}{\left(n^{2}-\varepsilon^{2}\right)} \\
& =\frac{1}{1-\varepsilon^{2}}+\sum_{n=2}^{\infty} \frac{\Delta n}{\left(n^{2}-\varepsilon^{2}\right)} \\
& <\frac{1}{1-\varepsilon^{2}}+\int_{1}^{\infty} \frac{d n}{\left(n^{2}-\varepsilon^{2}\right)} \\
& =\frac{1}{1-\varepsilon^{2}}+\frac{1}{2 \varepsilon} \ln \left(\frac{1+\varepsilon}{1-\varepsilon}\right)
\end{aligned}
$$

which is finite. Thus, the original series $s(y, z ; d)$ is convergent.

\section{REFERENCES}

Antiochos, S. K. 1987, ApJ, 312, 886

Antiochos, S. K. 1990, Mem. Soc. Astron. Ital., 61, 369

Antiochos, S. K. 1996, ASP Conf. Series, 95, 1

Antiochos, S. K. \& Dahlburg, R. B., 1997, Sol. Phys., 174, 5

Antiochos, S. K., DeVore, C. R., \& Klimchuk, J. A. 1999, ApJ, 510, 485

Antiochos, S. K., Karpen, J. T., \& DeVore, C. R. 2002, ApJ, 575, 578

Bateman, G. 1978, MHD Instabilities (MIT Press: Cambridge MA)

Bhattacharjee, A., Huang, Y-M, Yang, H., \& Rogers, B. 2009, Phys Plasmas 16, 112102, DOI:10.1063/1.3264103

Birn, J., Hesse, M., \& Schindler, K. 1998, J. Geophys. Res., 103, A4, 6843 
Biskamp, D. 1993, Nonlinear Magnetohydrodynamics (Cambridge University Press: New York)

Cassak, P., Shay, M. A., \& Drake, J. F. 2005, Phys. Rev. Lett., 95, 235002

Dahlburg, R. B., Klimchuk, J. A., \& Antiochos, S. K. 2003, Adv. Space Res., 32, 1029

Dahlburg, R. B., Klimchuk, J. A., \& Antiochos, S. K. 2005, ApJ, 622, 1191

Dahlburg, R. B., Klimchuk, J. A., \& Antiochos, S. K. 2006, Adv. Space Res., 37, 1342

DeVore, C. R. \& Antiochos, S. K., 2008, ApJ, 680, 740

Drake, J. F., Swisdak, M., Che, H., \& Shay, M. A., 2006, Nature, 443, 553

Edmondson, J. K., Lynch, B. L., Antiochos, S. K., DeVore, C. R., \& Zurbuchen, T. H. 2009, ApJ, 707, 1427

Edmondson, J. K., Antiochos, S. K., DeVore, C. R., Lynch, B. L., \& Zurbuchen, T. H. 2010, ApJ (submitted)

Furth, H. P., Killen, I., \& Rosenbluth, M. N. 1963, Phys. Fluids, 6, 459

Karpen, J. T., Antiochos, S. K., \& DeVore, C. R. 1995, ApJ, 450, 422

Karpen, J. T., Antiochos, S. K., \& DeVore, C. R. 1996, ApJ, 460, L73

Karpen, J. T., Antiochos, S. K., DeVore, C. R., \& Golub, L. 1998, ApJ, 495, 491

Klimchuck, J. A. 2006, Sol. Phys., 234, 41

Kulsrud, R. M. 2001, Earth Planets Space, 53, 417

Kulsrud, R. M. 2005, Plasma Physics for Astrophysics (Princeton University Press: Princeton)

Lynch, B. J., Antiochos, S. K., DeVore, C. R., Luhmann, J. G., \& Zurbuchen, T. H. 2008, ApJ, 683, 1192

Lynch, B. J., Antiochos, S. K., Li, Y., Luhmann, J. G., \& DeVore, C. R. 2009, ApJ, 697, 1918 
Miller, J. A., Cargill, P. J., Emslie, A. G., Holman, G. D., Dennis, B. R., La Rosa, T. N., Winglee, R. M., Benka, S. G., \& Tsuneta, S. 1997, J. Geophys. Res., 102, A7, 14631

Loureiro, N. F., Schekochihin, A. A., \& Cowley, S. C. 2007, Phys. Plasmas 14, 100703

Pariat, E., Antiochos, S. K., \& DeVore, C. R. 2009, ApJ, 691, 61

Parker, E. N. 1963, ApJS, 8, 177

Parker, E. N. 1972, ApJ, 174, 499

Parker, E. N. 1973, ApJ, 180, 247

Parker, E. N. 1983, ApJ, 264, 642

Parker, E. N. 1988, ApJ, 330, 474

Petschek, H. E. 1964, in The Physics of Solar Flares, NASA SP-50, ed. W. N. Hess (NASA: Washington), p. 425

Priest, E. R. 1981, Solar Flare Magnetohydrodynamics (Gordon and Breach: Newark)

Priest, E. \& Forbes, T. 2000, Magnetic Reconnection, (Cambridge University Press: New York)

Rappazzo, A. F., Velli, M., Einaudi, G., \& Dahlburg, R. B. 2008, ApJ, 677, 1348

Sweet, P. A. 1958, in Electromagnetic Phenomena in Cosmic Physics, ed. B. Lehnert (Cambridge University Press: New York), p. 123

Syrovatskii, S. I. 1971, Sov. Phys. JETP, 33, 933

Syrovatskii, S. I. 1978a, Astrophys. Space Sci., 56, 3

Syrovatskii, S. I. 1978b, Sol. Phys., 58, 89

Syrovatskii, S. I. 1981, ARA\&A, 19, 163

van Ballegooijen, A. A. 1985, ApJ 298, 421 
Welsch, B. T., DeVore, C. R., \& Antiochos, S. K. 2005, ApJ, 634, 1395

Yamada, M. 2007, Phys. Plasmas, 14, 058102

\begin{tabular}{|c|c|c|c|c|}
\hline Time $t$ & 100.1 & 111.66 & 115.74 & 147.64 \\
\hline Average Inflow Speed $V_{i}$ & 0.024 & 0.033 & 0.034 & 0.043 \\
\hline Average Outflow Speed $V_{o}$ & 0.237 & 0.302 & 0.278 & 0.349 \\
\hline Ratio $V_{i} / V_{o}$ & 0.102 & 0.109 & 0.122 & 0.123 \\
\hline
\end{tabular}

Table 1. Inflow and outflow speeds, averaged along and across the current sheet, and their ratio, for the four times shown in Figure 10.

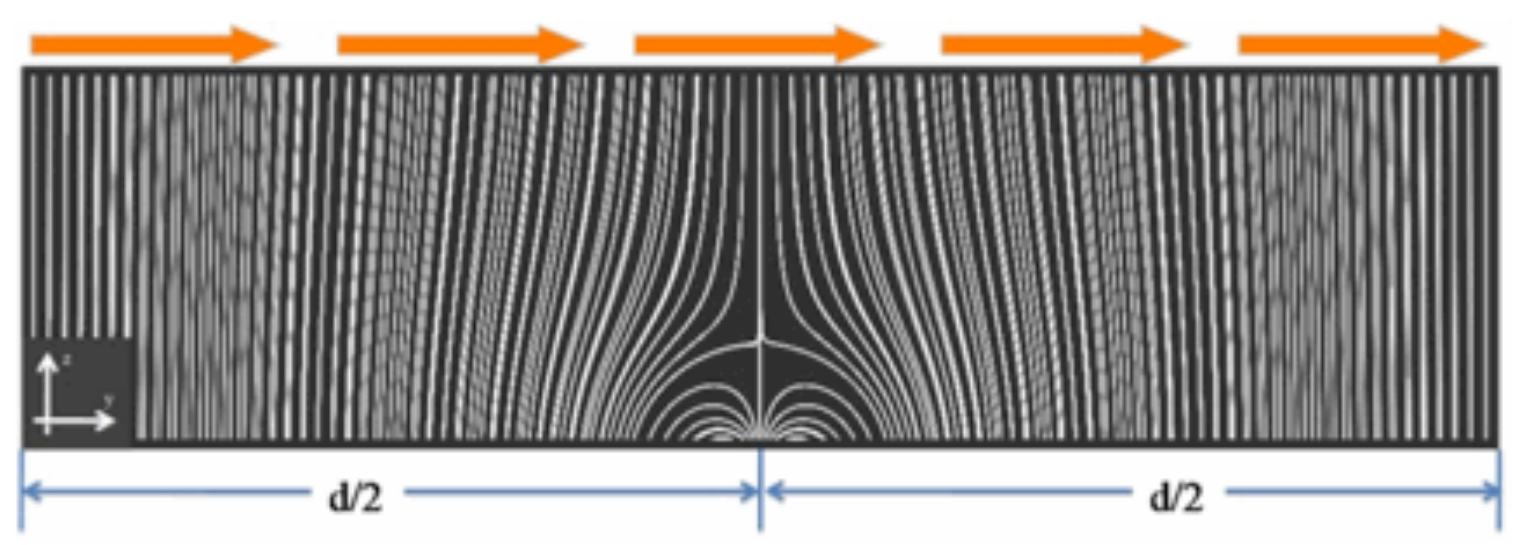

Figure 1. Initial magnetic field, consisting of a four-flux system in the $y z$ plane perpendicular to the translational symmetry axis $(x)$. A spatially uniform driving flow is imposed at the top boundary. 


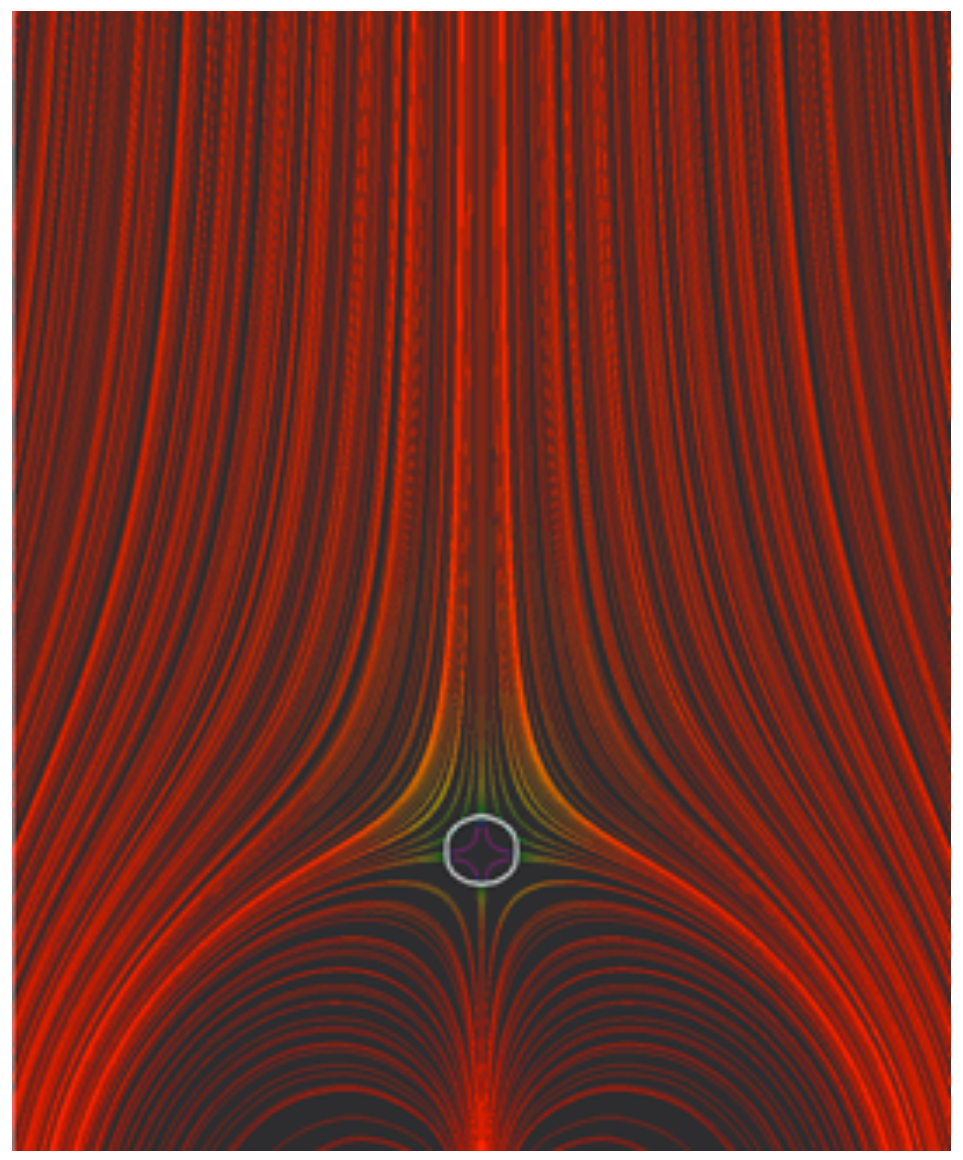

Figure 2. Plasma beta. Magnetic field lines are color-shaded according to the beta values along them: red is low, purple is high. The white contour marks the $\beta=1$ boundary.

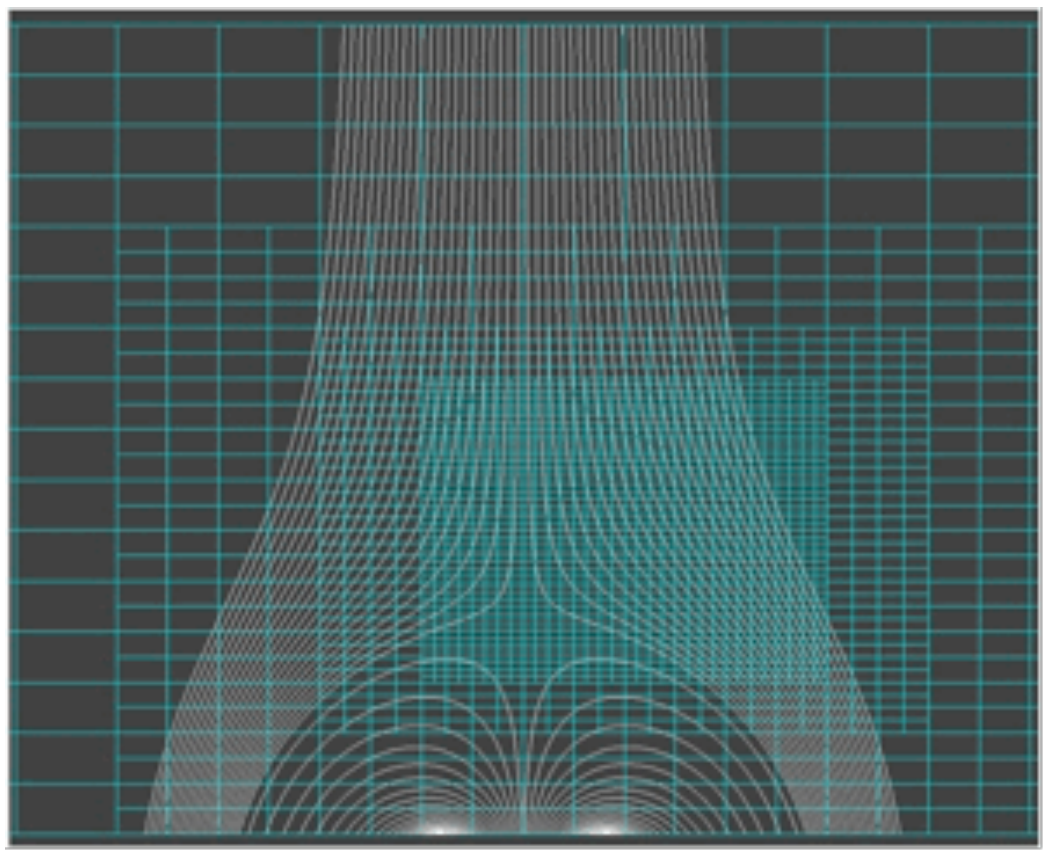


Figure 3. The initial grid in the perpendicular $(y z)$ plane. Grid lines (cyan) show every other grid cell, up to refinement level 6 (finest) encompassing the null line. The grid is invariant in the translationally symmetric $(x)$ direction.

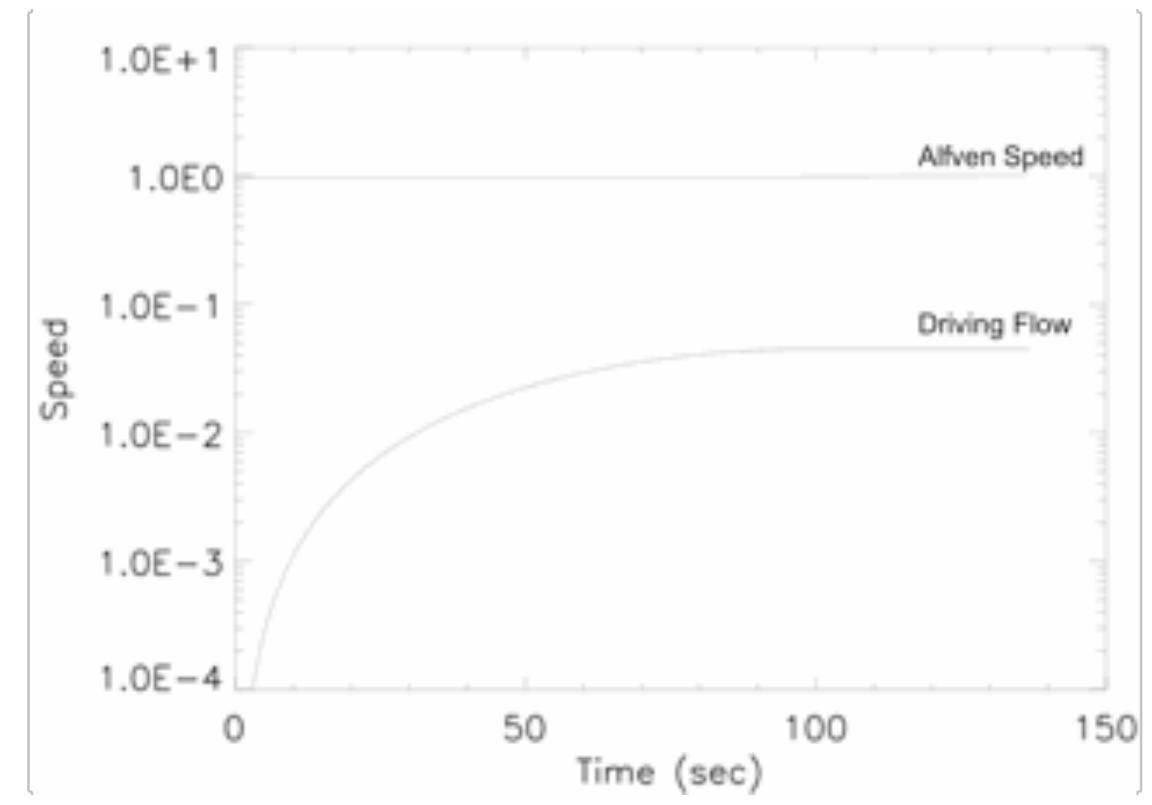

Figure 4. Time evolution of the driving flow speed and, for comparison, the initial global average Alfvén speed. 


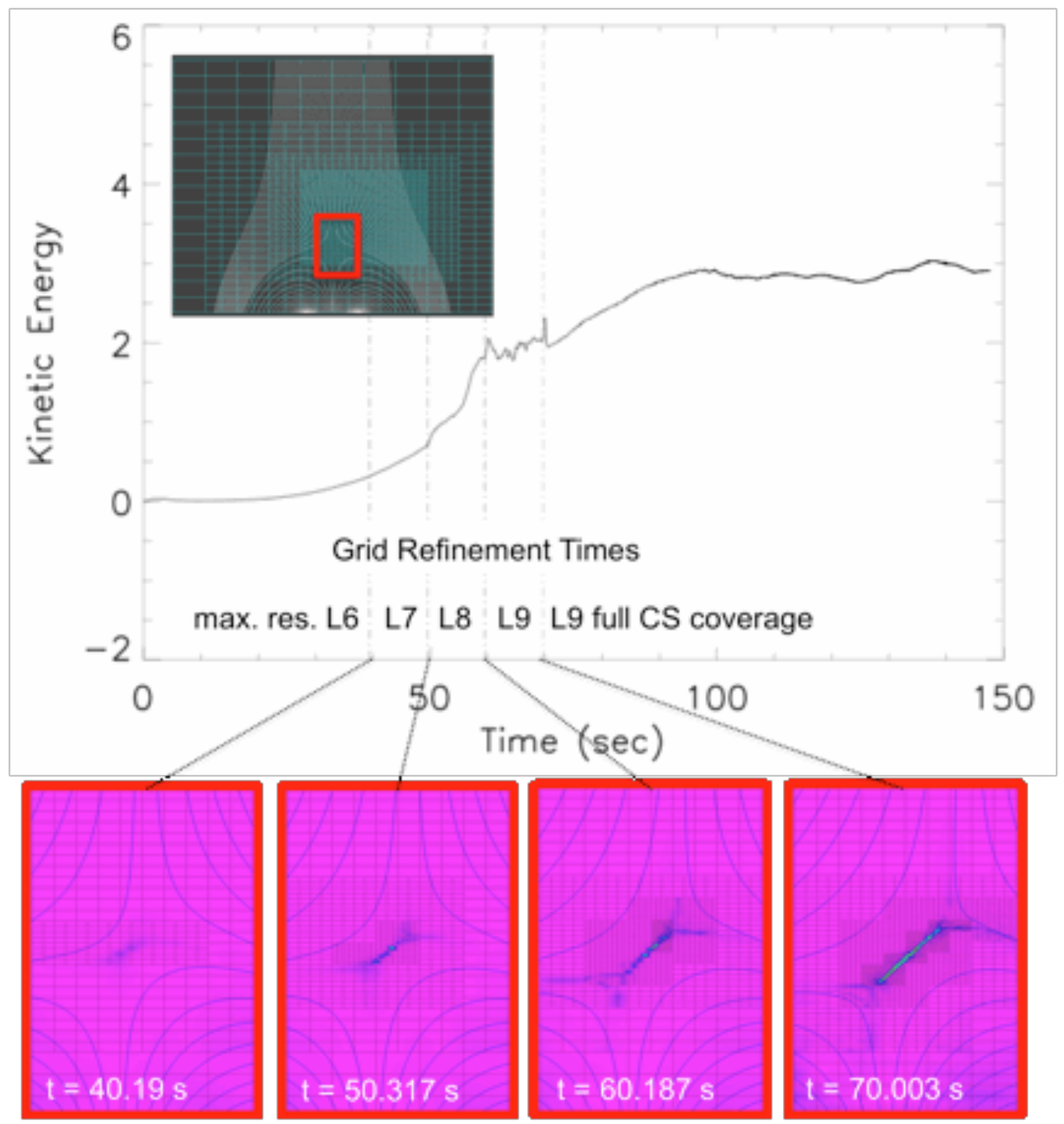

Figure 5. Time evolution of integrated kinetic energy (top) and of current-sheet intensity and extent, along with grid refinement (bottom). The adaptive mesh refinement refines the grid from level 6 to levels 7 through 9 at times $t=40.19,50.317$, and 60.187, respectively, and reaches full current-sheet coverage at the maximum allowed refinement level 9 for $t \geq 70.003$. Current-density magnitude is color-shaded in the bottom panels, which are zoomed views of the initial magnetic null line (the red rectangle in the inset figure, top). 

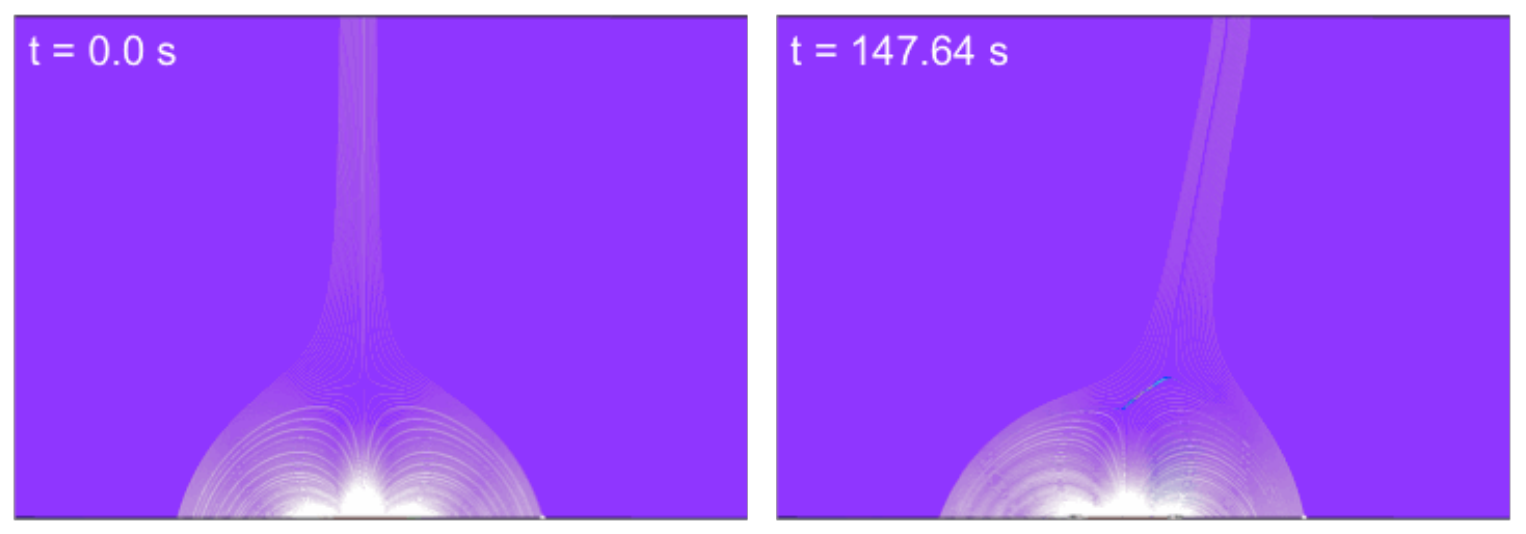

Figure 6. System configuration at the initial and final times $t=0.0$ and 147.64 seconds. Field lines are shown in white. The background color scale shows the current strength along the perpendicular direction. The singular current system is seen clearly between the dislocated spine lines. 

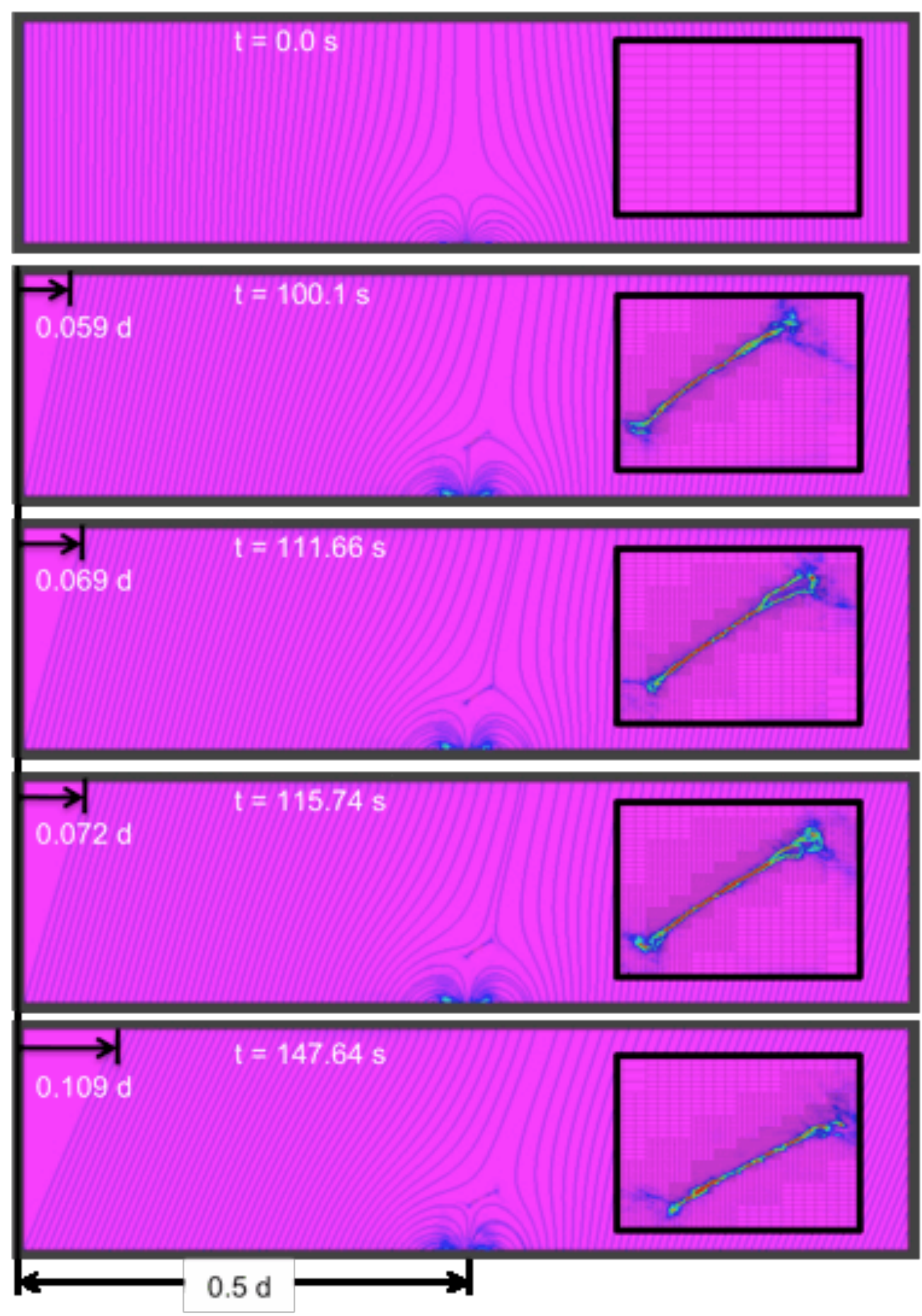

Figure 7. Global and current-sheet structure at times $t=\{0.0,100.1,111.66,115.74$, 147.64\}. The fully developed current sheet has little curvature and retains an overall length $L=0.04 d$. Current-density magnitude is color-shaded in each panel. 


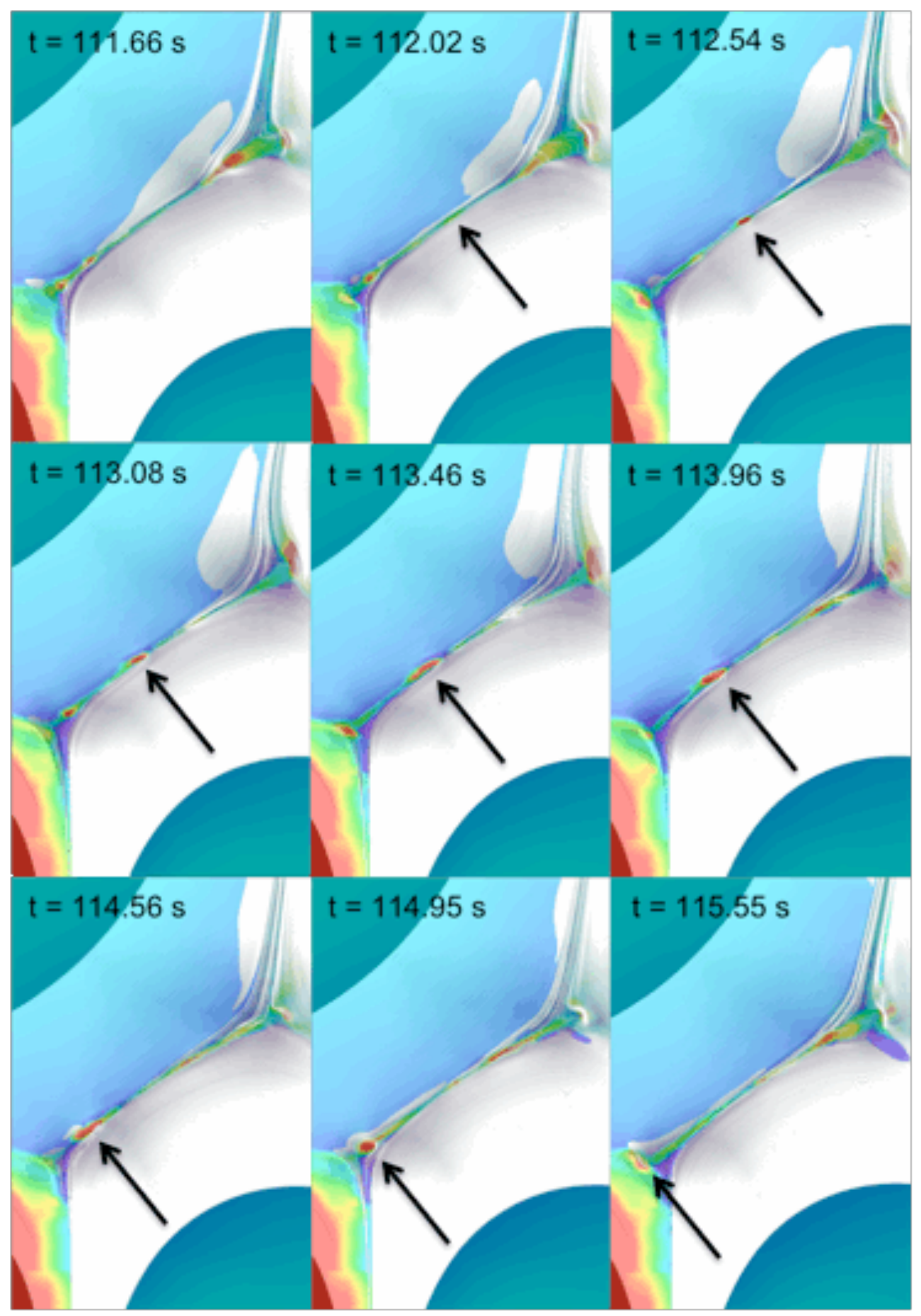

Figure 8. Time sequence showing the formation and ejection of a magnetic island (3D plasmoid cross-section), marked by the black arrows, in the perpendicular $y z$ plane at $x=$ 0 . Plasmoid formation through linear growth and nonlinear saturation occurs between times $t=111.66$ and 112.54, while plasmoid ejection occurs between times $t=113.08$ and 114.95 . 

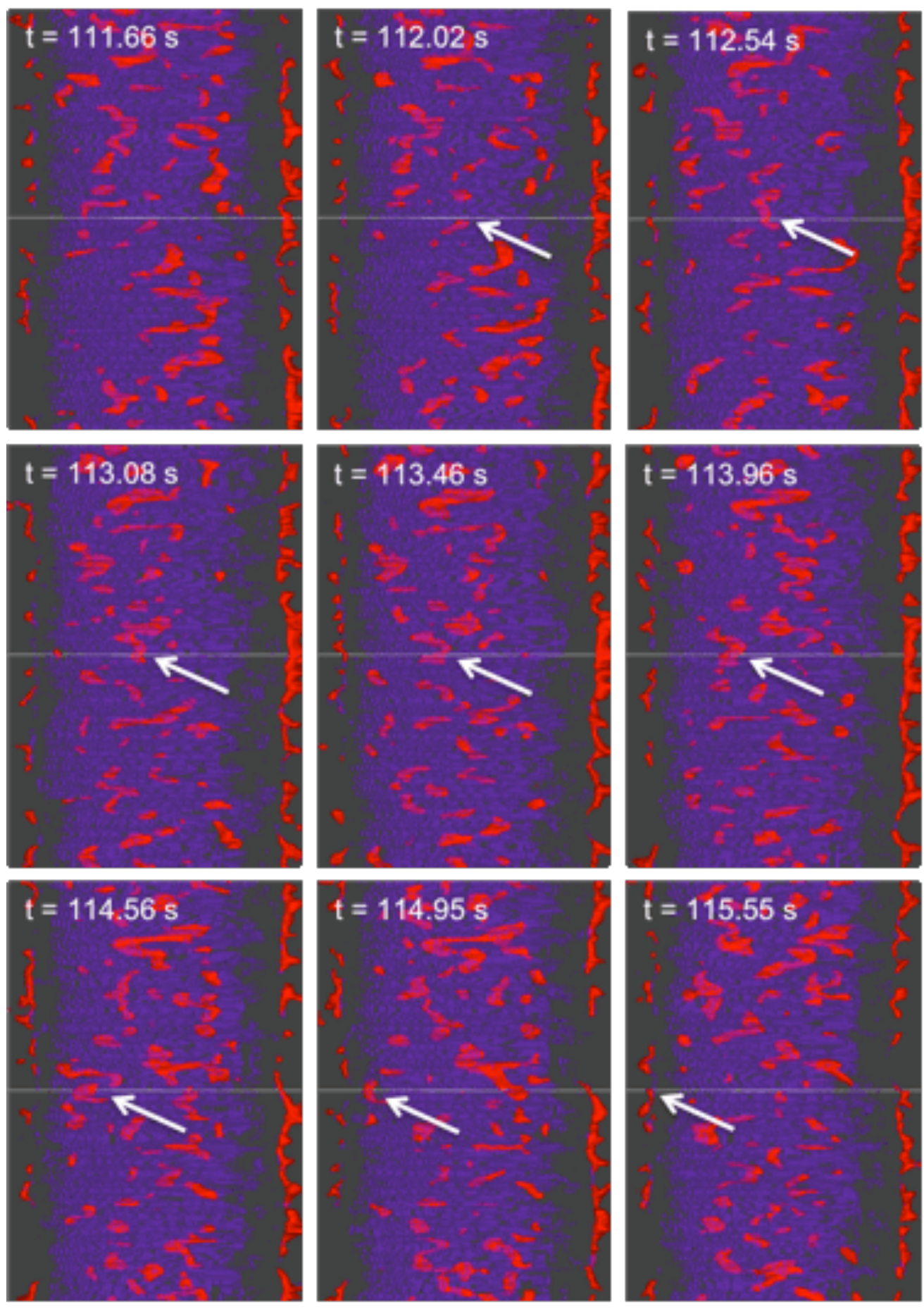

Figure 9. Saturated shading shows the 3D structure and evolution of dense plasmoids (red) in magnetic islands along the entire strong current-density structure (purple), in the $x y$ plane viewed as from above. The white arrows mark the plasmoid tracked in crosssection in Figure 8. 


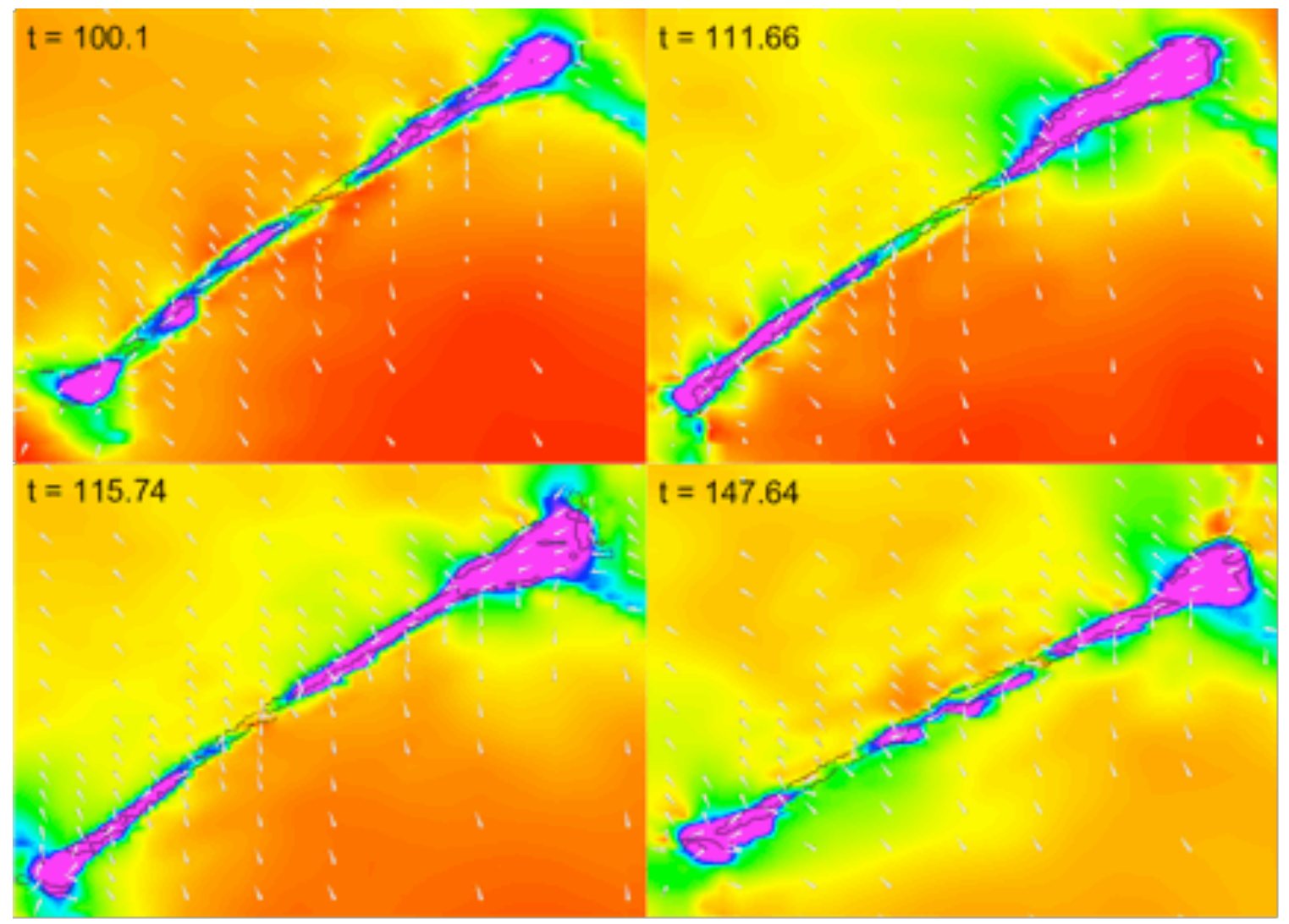

Figure 10. Velocity magnitude color-shaded at the mid-plane at times $t=\{100.1,111.66$, $115.74,147.64\} \mathrm{s}$. The reconnection jets are seen clearly as the purple shaded regions. Black contours are current-density magnitude, showing the approximate extent of the strong current sheet. White arrows mark the flow direction, showing the reconnection inflows from the side and the outflows at the ends of the current sheet. Note that the arrows are not scaled to the local velocity magnitude. 


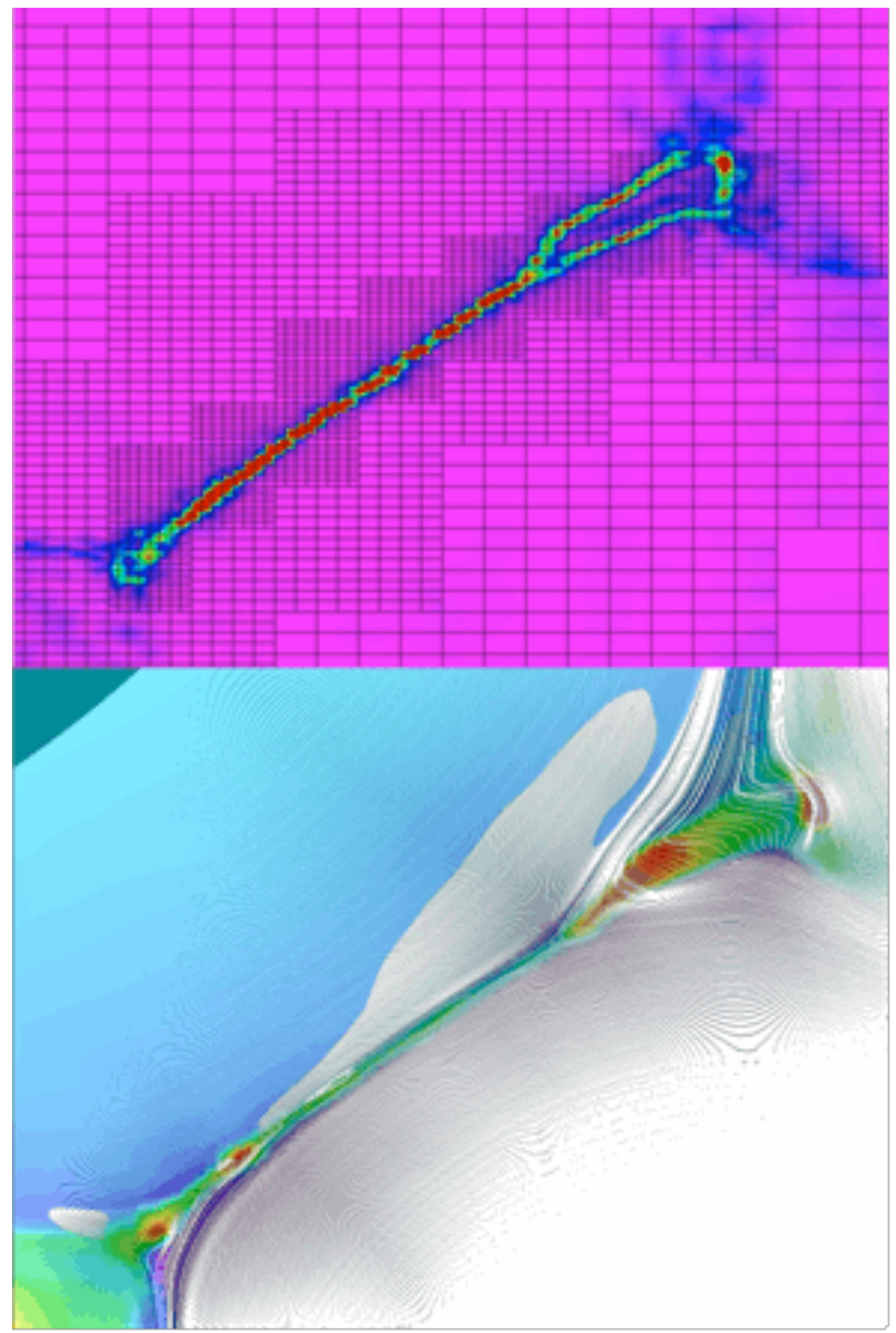

Figure 11. Evidence for a localized and transient Petschek-like geometry due to splitting of the current sheet at time $t=111.66$ by plasmoid ejection. Top: Color shading of current density, red signifying strong currents $(|J| \geq 5)$, with grid lines overlaid. Bottom: Color shading of plasma density, red signifying high densities $(\rho \geq 2)$, with field lines overlaid. The hard bends in the field lines at the right-hand reconnection jet are Alfvén waves associated with plasmoid ejection. 

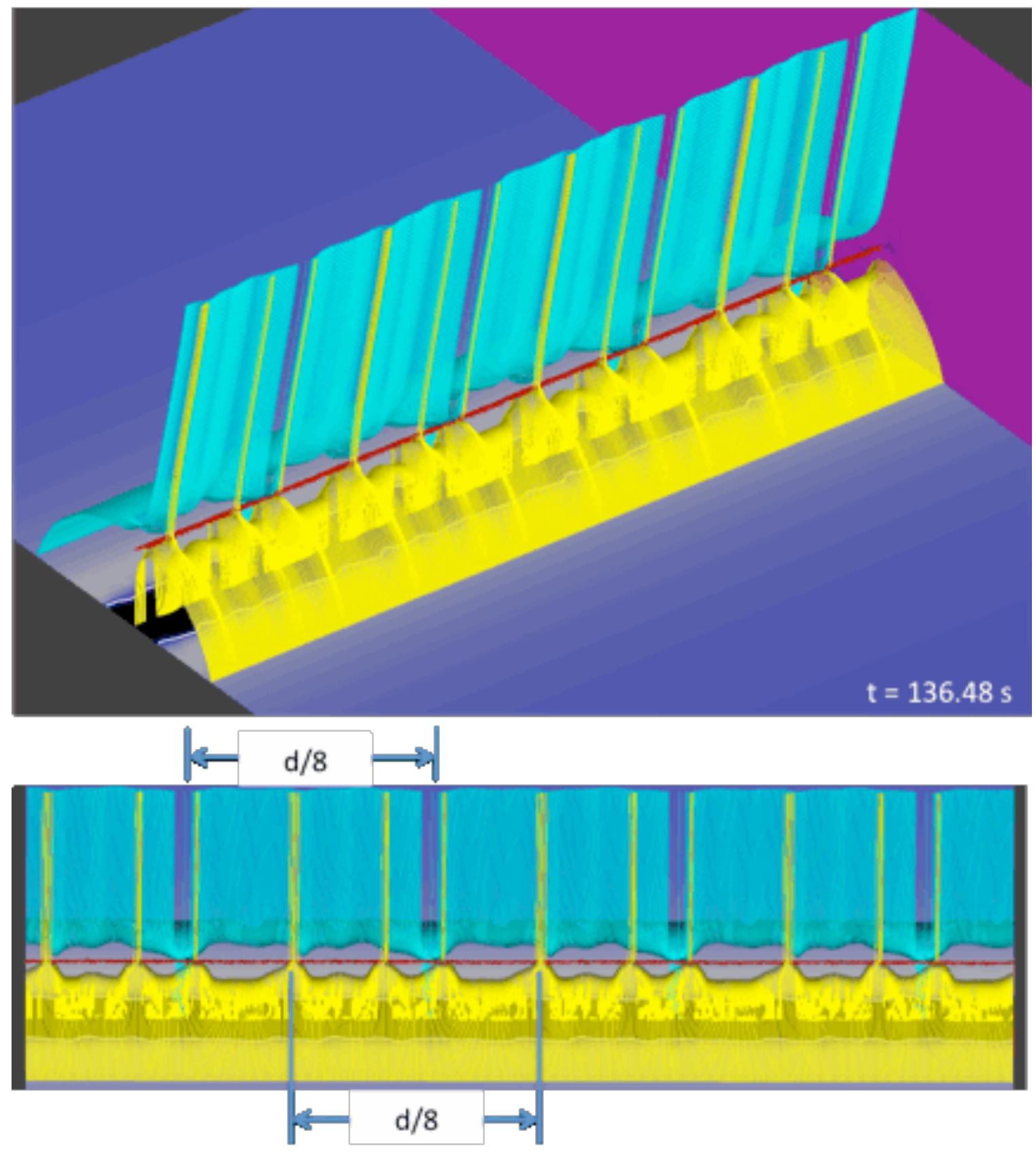

Figure 12. Large-scale periodic structure developing in the field lines along the third (initially axisymmetric) coordinate, exhibiting four wavelengths across the domain. 